\title{
Dynamics of Parasitaemia in Trypanosoma evansi Infection in Donkeys and the Efficacies of Buparvaquone and Isometamidium chloride Treatments
}

\author{
Garba $\mathrm{UM}^{1,}$, , Sackey $\mathrm{AKB}^{2}$, Lawal $\mathrm{AI}^{3}$, Esievo KAN ${ }^{4}$ \\ ${ }^{1}$ Veterinary Clinic, Equitation Department, Nigerian Defence Academy, Kaduna, Nigeria \\ ${ }^{2}$ Department of Veterinary Medicine, Faculty of Veterinary Medicine, Ahmadu Bello University, Zaria, Nigeria \\ ${ }^{3}$ Department of Veterinary Parasitology and Entomology, Faculty of Veterinary Medicine, Ahmadu Bello University, Zaria, Nigeria \\ ${ }^{4}$ Department of Veterinary Pathology, Faculty of Veterinary Medicine, Ahmadu Bello University, Zaria, Nigeria \\ Email address: \\ garbaum@yahoo.com (G. UM), akbsackeyabuzaria@yahoo.com (S. AKB), lawaidris@yahoo.com (L. AI), \\ kanescokok@yahoo.com (E. KAN) \\ ${ }^{*}$ Corresponding author
}

\section{To cite this article:}

Garba UM, Sackey AKB, Lawal AI, Esievo KAN. Dynamics of Parasitaemia in Trypanosoma evansi Infection in Donkeys and the Efficacies of Buparvaquone and Isometamidium chloride Treatments. Animal and Veterinary Sciences. Vol. 4, No. 3, 2016, pp. 32-38.

doi: 10.11648/j.avs.20160403.11

Received: May 27, 2015; Accepted: June 15, 2015; Published: May 23, 2016

\begin{abstract}
The aim of this study was to evaluate the efficacies of Buparvaquone (an anti-theilerial drug) and Isometamidium chloride (a standard trypanocide) in the treatment of experimental Trypanosoma evansi infection in donkeys. Out of a total of 24 donkeys, 6 were assigned to each of the experimental groups $A_{1}, A_{2}, A_{3}$ and $B$ at random. About 2.0x10 $10^{6}$ T. evansi parasite in $2 \mathrm{~mL}$ inoculum was used to infect each of the donkeys in groups $A_{1}, A_{2}$ and $A_{3}$ through jugular vein while group $B$ remained as Un-infected control. On day 28 post-infection, animals in groups $\mathrm{A}_{2}$ and $\mathrm{A}_{3}$ were treated with Isometamidium chloride and Buparvaquone respectively. Parasitaemia levels were evaluated post-infection and post-treatment using Haematocrit Centrifugation Technique and supported with mice inoculation tests to ascertain effectiveness of treatments. Group means of parasitaemia were presented on graphs. Result showed that parasites were first detectable from peripheral blood of the infected animals from day 3 and by day 4 post-infection all animals were parasitaemic and the levels fluctuated in subsequent days. The infected-untreated group of animals was aparasitaemic on day 100 post-infection onward using haematocrit centrifugation technique test but mice inoculation test detected relapse with death of all mice used due to heavy parasitemia. Similarly, the buparvaquone-treated group was aparasitemic on day 49 post-treatment (corresponding to day 77 post-infection) but mice inoculation test detected relapse of parasitaemia in the group and all mice used also died. Isometamidium-treated animals were negative for parasitaemia for 50 days on mice inoculation test but later on day 100 post-treatment, showed $60 \%$ relapse with parasiaemia level of $1^{+}$in the mice used and they did not die within 48 days of observation. In conclusion, experimental Trypanosoma evansi infection in donkeys have pre-patent period of 3-4 days, parasitaemia pattern is undulating and infecteduntreated animals became subclinical carriers from day 100 post-infection. The parasites in untreated, carrier animals maintained their virulence and pathogenicity. Buparvaquone on the other hand, depressed parasitaemia but the parasites were virulent and pathogenic to mice. Isometamidium chloride treatment resulted in incomplete clearance of parasitaemia due to Trypanosoma evansi and the relapsed parasites were avirulent and apathogenic. This is also the first report on the trial of buparvaquone for anti-Trypanosoma evansi effect in donkeys.
\end{abstract}

Keywords: Buparvaquone, Donkeys, Dynamics, Efficacies, Isometamidium chloride, Parasitaemia, Trypanosoma evansi

\section{Introduction}

In Nigeria, donkeys (Equus africanus asinus) are widespread especially in the northern region, where they serve as source of traction power in transport and ploughing for both pastoralists and settled farmers [1]. Coincidentally, 
T. evansi infection (surra) is endemic in donkeys in Nigeria [2]. The disease manifests as acute, in which case it is rapidly fatal in camels and horses or chronic form, which may persist for several months or years [3] as in donkeys which usually result in emaciation and death [4].

The causative agent; $T$. evansi belongs to the Salivaria section of pathogenic species of Trypanosoma and the subgenus, Trypanozoon which consists of three major trypanosome species; Trypanosoma brucei, T. evansi and T. equiperdum $[5,6]$.

The transmission of $T$. evansi among animals is mechanical, by biting flies mainly; Tabanus sp, and Stomoxys $s p$, Haematopota sp, Lyperosia sp and Chrysops sp [7]. Transplacental transmission mode has also been reported in donkeys [8].

The control of $T$. evansi infection in animals depends on chemotherapy and chemoprophylaxis relying on diminazene aceturate, quinapyramine, cymelarsan and suramin sodium, but emerging resistance of $T$. evansi strains to available drugs has limited the success of treatments $[9,10]$.

As part of efforts in the search for drugs with trypanocidal effects, the efficacies of buparvaquone and Isometamidium chloride against experimental T. evansi infection in donkeys were evaluated in this study. Buparvaquone is a secondgeneration injectable hydroxynaphthoquinone compound in aqueous solution with both curative and prophylactic effects against the causative agents of Bovine Theileriosis; Theileria parva (T. parva), T. sergenti and $T$. annulata [11, 12]. Isometamidium chloride belongs to the same chemical group as homidium; the phenanthridine aromatic amidine compounds $[13,10]$.

\section{Materials and Methods}

\subsection{Location of Research}

The Experiment was conducted in the Department of Veterinary Parasitology and Entomology Research Pen, Faculty of Veterinary Medicine, Ahmadu Bello University, Samaru campus, Zaria, North-Western Nigeria. Main campus, Samaru, Zaria, North-Western Nigeria. Main campus in Samaru is located on latitude $11^{\circ} 15^{\prime} \mathrm{N}$ to $11^{\circ} 3^{\prime} \mathrm{N}$ of the equator and longitude $7^{\circ} 30^{\prime} \mathrm{E}$ to $7^{\circ} 45^{\prime} \mathrm{E}$ of Greenwich Meridian [14].

\subsection{Experimental Animals}

Twenty four apparently healthy donkeys were purchased from International Livestock Market in Maigatari, Jigawa State, North-Western Nigeria and used for this experiment. The donkeys comprised of 12 males and 12 females, between the ages of 11 to 15 months old. The donkeys' ages were estimated at point of purchase using dental eruption pattern of the incisors teeth as described by Wayne and Melvin [15] and Joe [16].

Four (4) apparently healthy and haemoparasites-free, adult albino Wistar rats, with body weight range of $200 \mathrm{~g}-220 \mathrm{~g}$ were used as donors for the propagation of T. evansi to obtain sufficient inoculum for the experimental infection.

Mice inoculation tests (MIT) were conducted using apparently healthy and haemoparasites-free, adult Wistar mice (100 only) with body weight range of $14 \mathrm{~g}-16 \mathrm{~g}$. Two mice were used for each donkey screened, each time, as support test for HCT at pre-infection phase to ensure that they were haemoparasites-free, post-infection and posttreatment to determine relapse, ascertain effectiveness or otherwise of the treatment drugs against $T$. evansi infection. The rats and mice were sourced in batches from Animal Laboratory, Faculty of Pharmaceutical Sciences, Ahmadu Bello University, Zaria.

\subsection{Pre-infection Evaluation and Management of Experimental Animals}

\subsubsection{Housing and Acclimatisation}

On arrival in the Department of Veterinary Parasitology and Entomology, A.B.U., Zaria, the experimental donkeys were further examined for signs of disease and external parasites infestations. Topical sweat-resistant fly-repellant spray (Endure ${ }^{\circledR}$, Farnam co. inc., USA) was applied on the animals to keep flies away and then housed in prepared, flyproof experimental animal pen of the department. Wood shaving was used as bedding material on the floor of the pen; it was mucked daily to remove faecal droppings and changed as soon as the old one was bad. The experimental animals were acclimatised for 14 days [17].

In addition the following samples were collected and examined:

\subsubsection{Faecal Sample}

About $5 \mathrm{~g}$ of fecal sample was collected from the rectum of each animal and examined for gastro-intestinal parasites using floatation and sedimentation methods as reported by Charles [18]. The animals were dewormed using Fenbendazole bolus $\left(\right.$ Fenacure $^{\circledR}$, Ashish Life Sciences PVT ltd, India) at the dose rate of $10 \mathrm{mg} / \mathrm{kg}$ bd wt orally, once [19].

\subsubsection{Blood Sample}

Four millilitres $(4 \mathrm{ml})$ of blood was collected from each animal through the jugular vein using $5 \mathrm{ml}$ syringe and $18 \mathrm{G}$ needle. Three milliliters of the blood was placed in blood sample bottle containing liquid tripotassium salt of Ethylene Diamine Tetra-acetate (EDTA $\left.+\mathrm{K}_{3}\right), 1 \mathrm{mg} / \mathrm{mL}$ of whole blood as anticoagulant [20] and examined immediately for haemoparasites infections using 2 preparations of Wet Blood Film at 40x magnification, HCT and Giemsa Stained thin blood smear as reported by Weiser [21]. An average of 20 microscope fields of the stained thin smear slide was examined.

\subsubsection{Animal Inoculation}

The balance of $1 \mathrm{~mL}$ of blood collected from each donkey was transferred into sodium heparin-coated $1 \mathrm{~mL}$ sterile disposable syringe with $29 \mathrm{G}_{\mathrm{x}} 1 / 2$ inch needle and $0.5 \mathrm{~mL}$ of the blood was injected into a mouse intra-peritoneally [17] using 2 representative mice per donkey [3]. The inoculated 
mice were monitored for haemoparasites on HCT and Wet Film using tail tip blood at 48 hours intervals for 14 days [22], and remained under observation up to 60 days postinoculation.

\subsubsection{Feeding of Experimental Animals}

The experimental donkeys were provided with feed daily equivalent to $5 \%$ of their mean body weight, in the forms of; Sorghum/Maize stovers and Rhodes grass/groundnuts hay (4:1 ratio), the concentrate feed consisted of a mixture of sorghum grain $(500 \mathrm{~g})$, dried groundnut cake $(130 \mathrm{~g})$ and Maize/Wheat bran $(2.5 \mathrm{~kg})$ per $200-300 \mathrm{~kg}$ bd wt of animal. Salt lick blocks, rich in dicalcium phosphate and clean drinking water were provided ad-libitum as recommended by Aganga et al [23].

The albino rats and Wister mice were maintained in separate, labeled cages and were provided with commercially obtained pelletised feed in feeders and clean drinking water in sipper tubes ad libitum [17].

\subsection{Identification and Grouping of Animals}

Serially numbered aluminum plates (about 5g) neck-tags were assigned to the donkeys at random for identification. A sample frame of each gender was prepared and treated one after the other by random picking of animals' numbers and placing in experimental groups $A_{1}, A_{2}, A_{3}$ and $B$ to obtain a total of 6 animals ( 3 male and 3 female) in each experimental group. Choice of groups of animals inoculated with $T$. evansi as well as assigning of either Isometamidium chloride or Buparvaquone for group treatment was made at random [24].

The rats and mice were caged according to the group of donkeys they represented. Marks were applied on the various body parts of the rats and mice using permanent marker to reflect the donkey they represented.

\subsection{Propagation of Trypanosoma evansi}

Trypanosoma evansi (Sokoto isolate) originally isolated from camel and maintained in Wistar rats in the Department of Veterinary Parasitology and Entomology, Ahmadu Bello University, Zaria, was used in this experiment.

The level of parasitemia of the infected Wistar rat was first determined to be +4 (representing greater than 20 parasites per field of microscope after examining 20 fields) on haematocrit centrifugation technique (HCT). One milliliter of parasitaemic blood was collected via ocular vein into heparinised sample bottle and diluted with $1 \mathrm{~mL}$ phosphate buffered saline glucose solution. About $0.5 \mathrm{~mL}$ of the inoculums was inoculated into each of a set of 4 donor rats, intra-peritoneally using sterile $1 \mathrm{~mL}$ disposable syringe, $25 \mathrm{G}_{\mathrm{X}}$ $1 \frac{1}{2}$ inch needle $[22,17]$.

The donor rats were monitored for parasitaemia levels at 48 hours intervals using Wet Film method. Twelve days post-inoculation, the 4 rats had attained between +2 to +4 (i.e 6-10 to $>20$ parasites per microscope field). All donor rats were bled and the blood was pooled into a sterile conical flask containing heparin sodium 20 Units $/ \mathrm{mL}$ of blood. A total of $15 \mathrm{~mL}$ of pooled blood was obtained and the parasitemia was re-evaluated using haemocytometer [20]. The blood was diluted with phosphate buffered saline glucose solution to obtain an inoculum containing $1.0 \times 10^{6}$ T. evansi /mL [22].

\subsection{Experimental Infection and Monitoring of Animals}

The prepared inoculum was immediately used to infect the experimental animals in groups $A_{1}, A_{2}$ and $A_{3}$ at the rate $2 \mathrm{~mL}\left(2.0 \times 10^{6}\right.$ T. evansi) per animal through the jugular vein and the day was considered as day0 of infection. Group B animals remained as Un-infected (control) group.

The infected animals were monitored for parasitaemia using ear vein blood. The blood samples were collected once daily from all groups of animals between day1 and day7 post-infection (PI). This was followed by blood collection twice per week between day 8 and day 28 PI. The samples were examined using HCT and References and further reading may be available for this article. To view references and further reading you must purchase this article. Giemsastained thin blood smears as reported by Elaine and Margi [20]. Levels of parasitemia were scored using the method of Woo [25].

\subsection{Treatment of Specific Infected Animals}

On day $28 \mathrm{PI}$, which coincided with the third wave of parasitemia as determined on HCT, animals in groups $\mathrm{A}_{2}$ and $\mathrm{A}_{3}$ were treated and the day of first treatment was considered as day 0 of treatment. Group $A_{1}$ was Infected but Untreated, $\mathrm{A}_{2}$ was Infected and treated with Isometamidium (Securidium $^{\circledR}$, Laprovet, France) $1 \%$ solution, $0.5 \mathrm{mg} / \mathrm{kg}$ bd wt by deep intramuscular injection once [26] while group $\mathrm{A}_{3}$ was infected and treated with Buparvaquone (Butalex ${ }^{\circledR}$, Coopers K-brand Ltd, Kenya) $5 \% \mathrm{w} / \mathrm{v}, 2.5 \mathrm{mg} / \mathrm{kg}$ bd wt by deep intramuscular injection twice at 72 hours apart as recommended by the manufacturers. Animals in group B were uninfected and untreated (Control)

\subsubsection{Post-Treatment Monitoring}

The experimental donkeys were monitored further for levels of parasitemia for 148 days during which blood samples were collected from all animal groups as follows; once daily between dayl and day 7 post-treatment (PT), twice per week between day 8 and day 28 PT and once per week between day 29 and day 148 PT. The levels of parasitemia were evaluated on HCT and Giemsa-stained thin blood smear and were supported with mice inoculation test.

\subsubsection{Animal Inoculation}

Mice inoculation test (MIT) was performed for infecteduntreated in chronic stage of infection and for the infected, treated groups of donkeys post-treatment as a support for direct microscopic methods in-order to detect any case of subclinical infection. After 10 days or more of sustained aparasitemia from HCT in a group of donkeys, $1 \mathrm{~mL}$ of blood was collected from each donkey in the group through jugular vein using heparin-coated $2 \mathrm{~mL}$ syringe $19 \mathrm{Gx} 1 \frac{1}{2}$ inch needle. 
Half millilitre $(0.5 \mathrm{~mL})$ of collected blood was sub-inoculated into mouse intra-peritoneally using sterile $27 \mathrm{G}$ needles and 2 representative mice per donkey. The mice were identified and monitored for parasitemia using tail blood on HCT and Wet Film at 48 hours intervals [3]. Based the observed aparasitaemic status by groups of animals, MIT was conducted as follows;

Group $A_{1}$ (Infected-Untreated) Donkeys: Blood samples were collected from the donkeys in the group on day $110 \mathrm{PI}$ and sub-inoculated into mice.

Group $\mathrm{A}_{2}$ (Infected, Isometamidium chloride-treated) Donkeys: Mice inoculation test was performed for the group on days 21, 50 and 100 PT.

Group $A_{3}$ (Infected, Buparvaquone-treated) Donkeys:
Mice inoculation test was conducted for the group on day 59 PT.

\section{Data Analysis}

Group means of parasitaemia levels in the various stages of the experiment were determined and graphs were prepared for post-infection and post-treatment phases of the experiment using Microsoft Excel 2010 [27].

\section{Results}

The infection day 0 , prepatent-period, undulating parasitaemia and treatment day $28 \mathrm{PI}$ are as presented in Fig. 1.

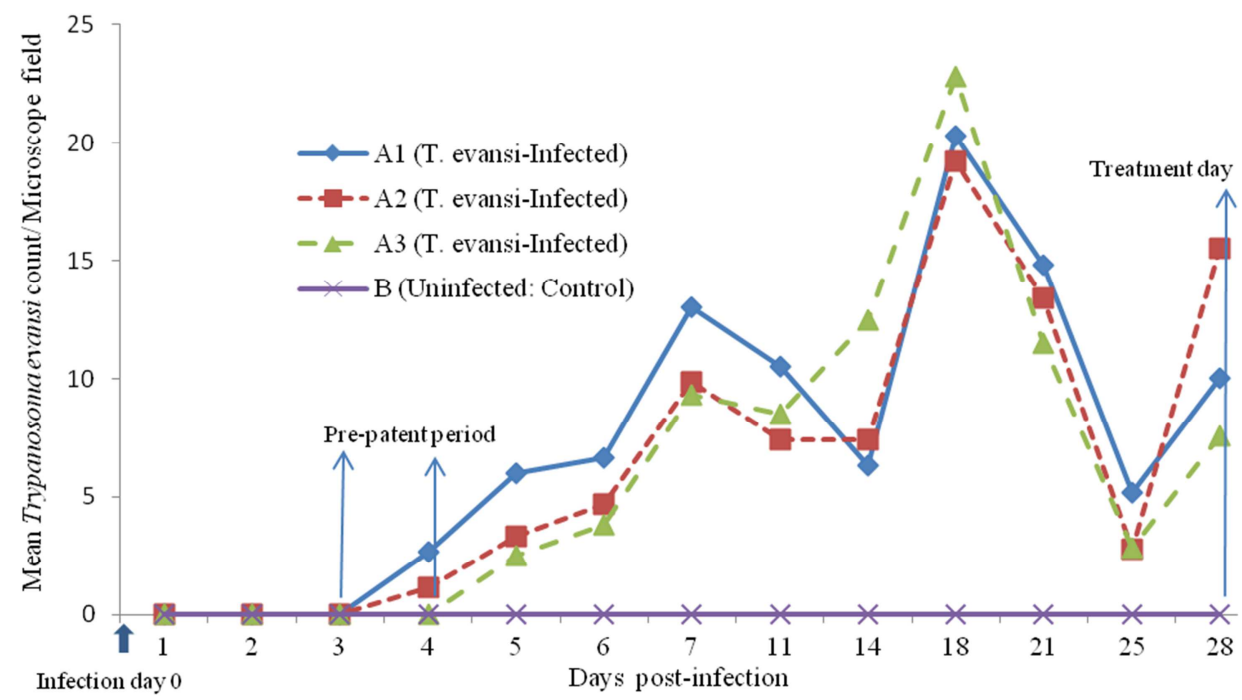

Fig. 1. Mean levels of parasitaemia in donkey experimentally infected with Trypanosoma evansi.

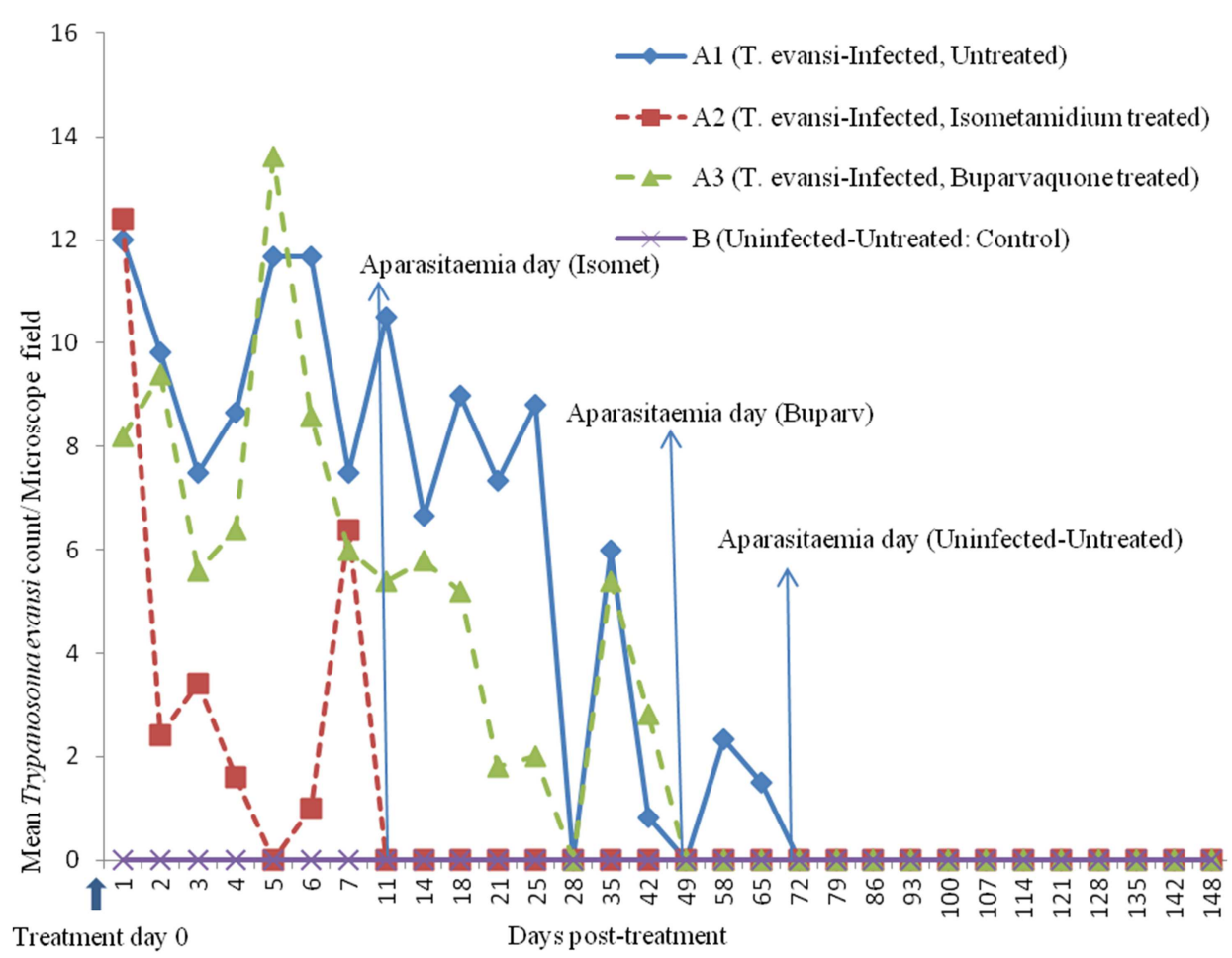

Fig. 2. Mean levels of Trypanosoma evansi observed in experimentally infected donkeys post-treatment. 
Group $\mathrm{A}_{1}$ (Infected-Untreated)

The infected-untreated group of animals maintained an undulating wave of parasitaemia until it became aparasitaemic on HCT examination on day $100 \mathrm{PI}$, equivalent to day72 PT, (Fig. 2). Mice inoculation test (MIT) conducted on day 110 PI resulted in death of all mice, barely 15 days post-inoculation from heavy parasitemia $(+3$ and $+4 /$ field $)$ on HCT and Wet Film (WF).

Group $\mathrm{A}_{2}$ (Infected, Isometamidium chloride-treated)

The $\mathrm{T}$. evansi-infected, Isometamidium chloride-treated group of animals had sharp drop in mean parasitaemia on HCT from approximately 12 /field on day1 to about 2 /field on day 2 PT and they became aparasitaemic on day 11 PT (Fig. 2). Mice inoculation tests on days 21 and 50 were negative but became positive on day 100 PT in 6/10(60\%) of mice used representing $3 / 5(60 \%)$ donkeys in the group. The test (MIT) showed that there was no detectable parasitaemia within 50 days PT and that by day $100 \mathrm{PT}$, subclinical status is detectable in $3 / 5(60 \%)$ of the donkeys treated with isometamidium. The inoculated mice showed only 1 parasite (1+) per microscope field both on Wet Film and HCT during 48 days of post-inoculation observation. The remaining 4 mice were negative both on WF and HCT for the same period of observation (Fig. 2).

Group $\mathrm{A}_{3}$ (Infected, Buparvaquone treated)

The Infected, Buparvaquone treated group became aparasitaemic on HCT on day 49 PT (Fig. 2). Following MIT on day $59 \mathrm{PT}$, all inoculated mice tested positive for T. evansi and died by day 23 post-inoculation observation.

\section{Discussion}

In this study, a pre-patent period (PP) of 3-4 days was observed and could be due to the level of pathogenicity of Sokoto isolate of T. evansi or susceptibility of the animal as determined by immunotolerance of donkeys to $\mathrm{T}$. evansi infection $[28,29]$.

The trend of parasitaemia in all groups of animals infected with $\mathrm{T}$. evansi was undulating suggesting trypanosomes ability to reside intermittently in intra- or extra-vascular fluids [30] and switches of surface glycoprotein in antigenic variation causing relapses of parasitaemia and remittent clinical signs $[31,32,33]$. The parasitaemia detected from Infected-Untreated group of animals using MIT and the death of all mice as a result of high level of parasitaemia means that untreated donkeys became chronic subclinical carriers of low level, virulent and pathogenic parasites from day 100 PI but detectable on MIT [3]. The negative parasitaemia status (on MIT) exhibited by Isometamidium-treated group of animals for 50 days PT with the later show of relapse on day 100 in $60 \%$ of treated animals which did not result in death of any of the inoculated mice suggest that Isometamidium chloride, controlled the T. evansi infection for 50 days as a standard trypanocides reported to have therapeutic and prophylactic effects against $T$. evansi infection for $2-4$ months in cattle $[34,35,19]$, that relapse can occur in $60 \%$ of treated donkeys from day 100 PT onward and that treatment renders the $\mathrm{T}$. evansi non-pathogenic and avirulent. This can be explained by the report that Isometamidium acts by cleavaging kDNA-topoisomerase complexes, resulting in the disintegration of minicircle network within $\mathrm{T}$. evansi kinetoplast [36] and mechanisms apart from those dependent on kDNA [37]. The show of aparasitaemic status by buparvaquone-treated group earlier than the InfectedUntreated group on HCT, with positive detection when MIT was used and death of all mice used for MIT from heavy parasitaemia suggest depression of parasitaemia due to buparvaquone treatment but the drug did not cure or clear infection and the donkeys were, therefore, chronic subclinical carriers of virulent and pathogenic T. evansi PI.

\section{Conclusion}

The dynamics of parasitemia in donkeys showed a prepatent period of 3 to 4 days and was charracterised by an undulating wave. The infected-untreated animals became chronic carriers with parasitaemia that was not detectable on HCT from day 100 PI onward but such parasites, when relapsed or detected using mice inoculation test, were virulent and pathogenic.

Isometamidium chloride $1 \%$, at $1 \mathrm{mg} / \mathrm{Kg}$ body weight, intramuscularly dosed did not completely clear T. evansi infection in donkeys. Relapse of upto $60 \%$ in Isometamidium-treated animals was detected from day 100 post-treatment using the mice inoculation test and the relapsed parasites were avirulent and non-pathogenic because they did not cause death of mice used for test.

Buparvaquone, $5 \mathrm{mg} / \mathrm{kg}$ body weight, intramuscularly dosed, twice at 3 days apart caused disappearance (depression) of parasitemia due to $\mathrm{T}$. evansi infection from peripheral circulation in donkeys but do not cure infection. The parasites were detected using the mice inoculation test and such parasites were virulent and pathogenic because they caused death of all mice used for test despite exposure to buparvaquone treatment. This is the first report on the trial of buparvaquone for anti-trypanocidal effect.

\section{Acknowledgements}

The financial contribution of Niger State Government under the Postgraduate Research Funding Programme is appreciated. Prof. Natala AJ, Head of Department of Veterinary Parasitology and Entomology approved the use of Research Animals Pen and protozoology laboratory. The technical staff of Veterinary Protozoology laboratory, Ahmadu Bello University, Zaria, assisted during laboratory tests.

\section{Ethical Considerations}

All procedures on the animals were performed as recommended by the European Union 'Directive 2010/63' contained in the report by Wolfensohn and Lloyd (2013) and 
approved by the Research and Ethics Committee of Ahmadu Bello University, Zaria, Nigeria.

\section{References}

[1] Blench R, de Jode A Gherzi E. Donkeys in Nigeria: history, distribution and productivity. A resource book of the Animal Traction Network for Eastern and Southern Africa (ATNESA). ACP-EU Technical Centre for Agricultural and Rural Cooperation (CTA), Wageningen, The Netherlands. 2013; 244p. ISBN 92-9081-219-2. Retrieved January 01, 2013, from http://www.atnesa.org

[2] Ilemobade AA. Studies on the incidence and pathogenicity of Trypanosoma evansi in Nigeria. 11: The pathogenicity of Trypanosoma evansi for equine and bovine species. ISCTRC, OAU/STRC. 1971; 105:107-114.

[3] OIE [Office International des Epizootes]. Trypanosoma evansi infection (surra).Version adopted by The World Assembly of Deligates of the OIE in May 2010. 2010; Chapter 2.1.17. Retrieved November 24, 2012 from, http://www.oie.int/fileadmin/Home/eng/Health_standards/tah m/2.01.17_TRYPANO.pdf

[4] Cadioli FA, Marques LC, Machado RZ, Alessi AC, Aquino LPCT, Baznabe PA. Experimental T. evansi infection in donkeys: Haematological, biochemical and histopathological changes. Arquivo Brasileiro de Medicina Veterinaria e Zootecnia. 2006. doi.org/10.1590/S0102-09352006000500008

[5] Kirsten Gillingwater aus Hornussen AG. Discovery of novel active diamidines as clinical candidates against Trypanosoma evansi infection, Inaugural dissertation, zur Erlangung der Würde einer Doktorin der Philosophie, vorgelegt der, Philosophisch-Naturwissenschaftlichen Fakultät, der Universität Basel. 2007; 229pp.

[6] WHO [World Health Organisation]. Trypanosomiasis, Human African (sleeping sickness). 2012; Fact sheet No: 259 Updated October 2012, Retrieved May 01, 2013 from, http://www.who.int/mediacentre/factsheets/fs259/en/.

[7] Luckins AG. Trypanosomiasis caused by Trypanosoma evansi in Indonesia. J Protozool Res. 1999; 144-152.

[8] Kumar R, Kumar S, Virmani N, Yadav SC. Transplacental transmission of Trypanosoma evansi from experimentally infected donkey mare to neonatal foal. J Equine Vet Sci. 2015; DOI: http://dx.doi.org/10.1016/j.jevs.2015.02.004

[9] Zhou J, Shen J, Liao D, Zhou Y, Lin J. Resistance to drug by different isolates of Trypanosoma evansi in China. Acta Tropica. 2015; 90: 271-275.

[10] FAO (Food and Agriculture Organisation). A field guide for the diagnosis, treatment and prevention of African animal trypanosomiasis. Retrieved September 22, 2012. From, www.//C:/Users/HPUSER/

Documents/trypanosomeschemotherapy.htm.

[11] Dhar S, Malhotra DV, Bhushan C, Gautam OP. Chemoprophylaxis with Buparvaquone against theileriosis in calves. Vet Record. 1987; 120: 375.

[12] Antti M, Tracy G, Jarkko R, Tapio N, Jouko V, Ari K, Simon LC, Tomi J. Synthesis, In Vitro evaluation and antileishmanial activity of water soluble pro-drugs of Buparvaquone. J Med Chem. 2004; 47(1): 188-195.
[13] Anene BM, Onah DN, Nawa Y. Drug resistance in pathogenic African trypanosomes: what hopes for the future? Vet Parasitol. 2001; 96: 83-100.

[14] Abbas II, Arigbede YA. Green area mapping of Ahmadu Bello University Main Campus, Zaria, Nigeria using remote sensing (Rs) and geographic information system (Gis) techniques. $J$ Geog Regional Plan. 2012; 5(10): 287-292. DOI: 10.5897/JGRP12.024.

[15] Wayne L, Melvin B. Determining age of horses by their teeth, Extension and Agricultural Information, 1-98 Agriculture Building, Columbia, MO 65211; XPLOR website:.http://extension.missouri.edu/publications/index.aspx . 2000. Accessed 12:30 GMT July 20, 2011.

[16] Joe A. The Donkey Sanctuary. British Veterinary Association. Retrieved February 11, 2012. From, www.bva.co.uk

[17] Wolfensohn S, Lloyd M. (2013). Handbook of Laboratory Animal Management and Welfare. Fourth edition, WileyBlackwell Publishing ltd, U.K., 371pp.

[18] Charles M. Internal parasites, External paraspites. In: Charles M.H. and Margi S. (Eds) Laboratory procedures for veterinary technicians. Fifth edition, Mosby Elsevier Inc., St Louis Missouri. 2007;.pp. 187-252.

[19] YO. Aliu, Nigerian Veterinary Formulary; Handbook of Essential Veterinary Drugs, Biologics and Pesticide Chemicals First edition, (Veterinary Council of Nigeria. 2007; p. 67.

[20] Elaine A, Margi S. Hematology and hemostasis. In: Charles M. H. and Margi S. (Eds) Laboratory Procedures for Veterinary Technicians. Fifth edition, Mosby Elsevier Inc., St Louis Missouri. 2007; pp. 27-73.

[21] Weiser G. Sample collection, processing and analysis of laboratory service options, In: Mary A. T., Glade W., Robin W. A. and Terry W. C. (Eds) Veterinary Hematology and Clinical Chemistry, second edition, Wiley-Black Well, U. K.. 2012; pp. 34-50.

[22] Monzon CM, Mancebo OA, Roux JP. Comparison between 6 parasitological methods for diagnosis of Trypanosoma evansi in the subtropical area of Argentina. Vet Parasitol. 1990; 36: 141-146.

[23] Aganga AA, Letso M, Aganga AO. Feeding donkeys, Livestock Research for Rural Development, 2000; 12(2): 2000. http://www.cipav.org.co/lrrd/lrrd12/2/agan122.htm. Retrieved on 12/07/12, 1200 GMT

[24] Aviva P, Poul W. Statistics for Veterinary and Animal Science. Third edition (Wiley-Blackwell ltd, UK. 2013; 391pp.

[25] Woo PTK. The haematocrit centrifugation technique for the diagnosis of African trypanosomiasis. Acta Tropica. (1970; 27 (4): $384-386$.

[26] Aliu YO. Nigerian Veterinary Formulary; Handbook of Essential Veterinary Drugs, Biologics and Pesticide Chemicals. First edition, Veterinary Council of Nigeria. 2007; p. 67.

[27] Mead R, Curnow RN, Hasted AM. Statistical Methods in Agriculture and Experimental Biology (Texts in Statistical Science). Third edition, Chapman and Hall/CRC, UK. 2002; 488pp. 
[28] Brun R, Hecker V, Lu V. Trypanosoma evansi and Trypanosoma equiperdum: Distribution, biology, treatment and phylogenetic relationship (A review), Vet Parasitol. 1998; 79(1998): 95-107.

[29] Herrera HM, Davila AM, Norek A, Abru UG, Souza SS, Andrea PSD, Jansen AM. Enzootiology of Trypanosoma evansi in the pantanal. Braz Vet Parasitol. 2004; 125: 263-275.

[30] Sudarto MW, Tabel H, Haines DM. Immunohistochemical demonstration of Trypanosoma evansi in tissues of experimentally infected rats and a naturally infected water buffalo (Bubalus bubalis). J Parasitol. 1990; 76 (2): 162-167.

[31] Ramirez LE, Wells EA, Betancourt A.. La trypanosomises en los animales domesticos en Colombia (S.L.) Centro International Agricultura Tropical. 1979; 71pp.

[32] Stephen L. Trypanosomiasis: A Veterinary Perspective. Pergamon Press, New York, USA. 1986.

[33] Hutchinson OC, Picozzi K., Jones NG, Mott H, Sharma R, Welburn SC, Carrington M. Variant Surface Glycoprotein gene repertoires in Trypanosoma brucei have diverged to become strain-specific. BioMed Central Genomics. 2007; 8: 234. doi:10.1186/1471-2164-8-234.

[34] Touratier L. The advantages of using Isometamidium for the control of animal trypanosomiasis. International Scientific Council for Trypanosomiasis Research and Control. Sixteenth meeting, Yaounde, Cameroon, 1979. OAU/STRC. 1981; 308316.

[35] Mira SF, Ralph R. Manual of Tropical Veterinary Parasitology. First edition, C.A.B., England. 1989; pp. 181260 .

[36] Shapiro TA, Englund PT. Selective cleavage of kinetoplast DNA minicircles promoted by antitrypanosomal drugs. Proceedings Natl Acad Sci USA. 1990; 87: 950-954.

[37] Kaminsky R, Schmid C, Lun ZR. Susceptibility of dyskinetoplastic Trypanosoma evansi and Trypanosoma equiperdum to isometamidium chloride. Parasitol Res. 1997; 83: 816-818. 